\title{
Shear Strength Parameters of Improved Peat by Chemical Stabilizer.
}

\begin{abstract}
The present research aimed to discuss the applicability of cationic grouts in geotechnical engineering. The effects of several cationic stabilizers such as monovalent (sodium silicate), divalent (calcium oxide and calcium chloride), and trivalent (aluminum hydroxide) were investigated on shear strength improvement of tropical peat samples. The unconfined compressive strength (UCS) tests were performed after the time frame of 7,21 , and 30 days as curing time, respectively. Apart from the physicochemical characteristics of the stabilized peat, scanning electron microscopy and energy-dispersive X-ray spectroscopy tests were also carried out to study the ongoing microstructural changes. It is to be noted that the shear strength values for peat samples rose to $8,6,6$, and $4 \%$ of sodium silicate, calcium oxide, calcium chloride, and aluminum hydroxide, respectively. The highest observed UCS outcome is the one taken from the calcium oxide where the UCS of treated peat after 30-day curing time increased to $76 \mathrm{kPa}$. The strength changes resulted from the various cationic stabilizers can best be explained via the consideration within the mineralogical composition as well as those physicochemical changes happening in the peat.
\end{abstract}

Keyword: Tropical peat, Soil improvement, Chemical grouts, Microstructure analysis 